\title{
Multi-parametric fit method in reconstruction of brachytherapy needles
}

\author{
Jeno Palvolgyi*
}

Petz County Teaching Hospital, Gyor, Department of Oncoradiology, Zrinyi str.13, H-9002 Gyor, Pf. 92, Hungary

Received 21 October 2011; received in revised form 29 March 2012; accepted 4 April 2012

Available online 12 May 2012

\section{KEYWORDS}

Brachytherapy;

Reconstruction;

Carcinoma of the

vulva $8753 \mathrm{Jw}$

\section{Introduction}

To prevent the applicator movement, the most sophisticated method in biplane projection image-based

* Tel.: +36 96 418244; fax: +3696433671.

E-mail address: palvolgyij@petz.gyor.hu.
Abstract Purpose: To adapt the multi-parametric fit method to reconstruct brachytherapy needles inserted with free hand technique for treatment of surface malignancies.

Methods: An alternative reconstruction method for brachytherapy (BT) needles is presented. The method is based on the digitized tip and end coordinates on pairs of posterior-anterior and posterior-oblique reconstruction images obtained with a non-isocentric $\mathrm{C}$-arm. The needles tip and end coordinates are computed with the multi-parametric fit method that also incorporates the determination of the magnification factors of the reconstruction images. We tested the reconstruction accuracy with radio-opaque markers inserted into known positions. The range of $\mathrm{C}$-arm angles that resulted in an accurate reconstruction was also investigated. We applied the method in treatments of the vulvar cancer using 3 to 5 pieces of BT needles located in a distance of approximately $1 \mathrm{~cm}$.

Results: The phantom test showed largest difference between the reconstructed and expected distance between the simulated needles $\pm 1.3 \mathrm{~mm}$. In vulvar insertions, the reconstructed position of the BT needles obtained with different image pairs agreed within $3 \mathrm{~mm}$.

Conclusion: The reconstruction accuracy of the multi-parametric fit method in with proper imaging is suitable for the clinical use.

(c) 2012 Associazione Italiana di Fisica Medica. Published by Elsevier Ltd. All rights reserved. applicator reconstruction is the use of integrated brachytherapy unit (IBU). If an IBU is not available, a C-arm fluoroscopy unit located in the BT room can be applied as a localizer. The majority of $\mathrm{C}$-arms are non-isocentric machines. Various methods have been published for application of such devices as BT localizer [1-5]. At our institution, we also introduced the use of a non-isocentric C-arm (Mobilgil AR7, Giraldoni, Italy) as a BT localizer in 
gynecological treatments using a variable angle reconstruction method. The applicator insertions are performed in the lithotomic position. After finishing the procedure, with patient in the same position, a pair of posterioranterior $\left(\mathrm{PA}=180^{\circ}\right.$ ) and posterior-oblique (PO) reconstruction images with the $\mathrm{C}$-arm located in $\mathrm{BT}$ treatment room are obtained. The video signal from the $\mathrm{C}$-arm monitor is frame grabbed and the images are converted to Dicom $^{\circledR}$ format. The magnification factor of images obtained with the non-isocentric machine varies with the $\mathrm{C}$ arm orbital angle. Since the $512 \times 512$ pixels images are calibrated: the pixel size is $0.32 \mathrm{~mm}$, the determination of the magnification factors is carried out with measurement of the magnified length of radio-opaque markers inserted into the tandem using a software ruler. After determination of the magnification factors, the catheter reconstruction and dose calculation are performed by a commercial treatment planning system.

We had previously developed a multi-parametric fit (MPF) method to reconstruct the Fletcher-SuitDelclos (FSD) applicator without the need of previous determination of the magnification factors [6]. The FSD applicator reconstruction with the MPF method relies on the known geometry of the tandem and the ovoid and on pairs of biplane projection images. The actual FSD applicator geometry is obtained by adaptation of the geometry of tandem and ovoid by translations and rotations using a simplex fit. The determination of the magnification factors of the reconstruction images is incorporated.

At our institution, BT of vulvar cancers is performed with insertion of 3-6 pieces of BT needles (Nucletron) into the inner part of the labia majora. The needles are inserted approximately parallel at a distance of $1 \mathrm{~cm}$ in a single plane. The patient lies on a treatment table (Easynox, France) in the lithotomic position. After finishing the insertion, the patient remains in the same position while the reconstruction images are taken with a $\mathrm{C}$-arm, located in the BT room. After preparation of the dose plan, the irradiation is performed.
In vulvar insertions, the inclination of the needles from the coordinate axis of the reconstruction images is usually large. For computation of correction of the magnification factor, the measurements of inclination angles are needed. Instead of this process, we aimed to adapt the multiparametric fit method to determine the magnification factors and to reconstruct the BT needles.

\section{Materials and methods}

The MPF reconstruction method is based on known geometry of a rigid applicator and biplane reconstruction images obtained by a C-arm. We prefer pairs of PA and PO, or pairs of two $P O$ reconstruction images instead of usual PA and lateral ones, because for two reasons: the image quality of PO images is better compared to lateral ones and it is more convenient to set the $\mathrm{C}$-arm to $\mathrm{PO}$ position, than to lateral one. Our method for use of a non-isocentric C-arm as BT localizer is summarized as follows and illustrated in Fig. 1. On the PA image we select a radio-opaque marker inserted into the needle. We shift the C-arm until the main beam axis intercepts the selected point and we obtain PA image. Before taking PO reconstruction image we compensate the main beam axis movement with the $\mathrm{C}$ arm angle. We shift the $\mathrm{C}$-arm laterally until the main beam axis intercepts the selected point on the PO image. Although, the two main beam axis intercept the same point on both images, this setup differs from the isocentric one: the magnification factors of the two reconstruction images are different and not known exactly. Despite of this difference, we refer the crossing point of the two main beam axis as isocenter.

To reconstruct the spatial position of the BT needles with incorporation of the determination of the magnification factors of the reconstruction images, we adapted the MPF method as follows. We suppose that the BT needles are strait. We digitize two points per needles on each reconstruction images with the ImageJ v1.24 program (http:// rsb.info.nih.gov/ij) and we save theirs coordinates. These

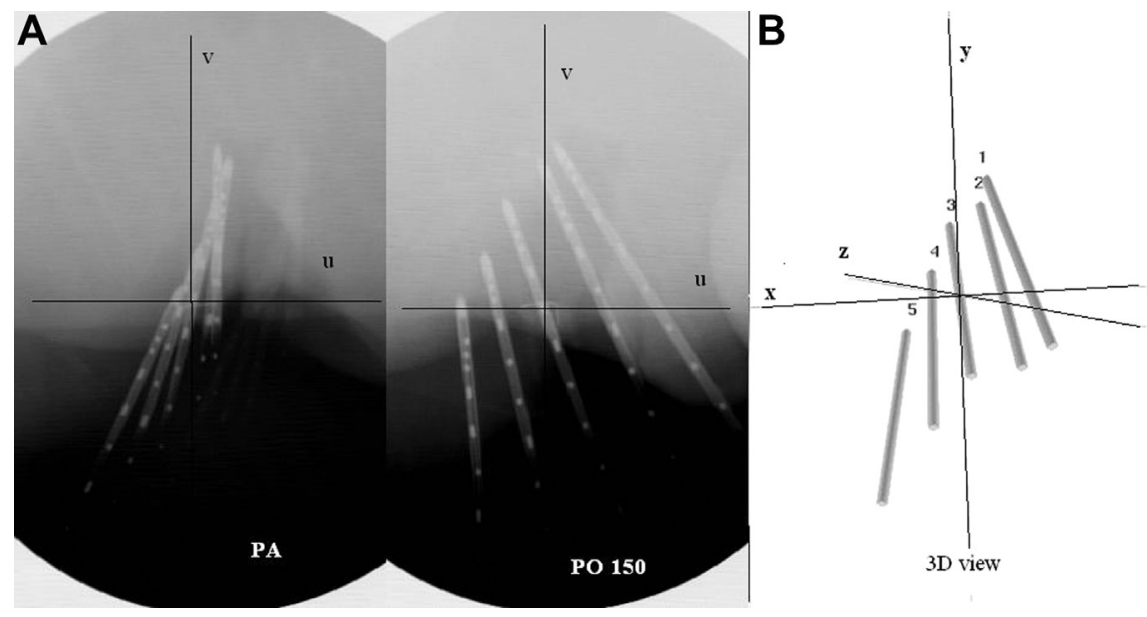

Figure 1 (A) Pairs of reconstruction images obtained by a mobile C-arm at $180^{\circ}$ (PA) and at $150^{\circ}$ (PO). On the PA image, we selected the point: needle \#3, third radio-opaque marker from the tip. We shifted the $\mathrm{C}$-arm until the main beam axis intercepts the selected point on both images. (B) The three-dimensional view of the reconstructed needles. 


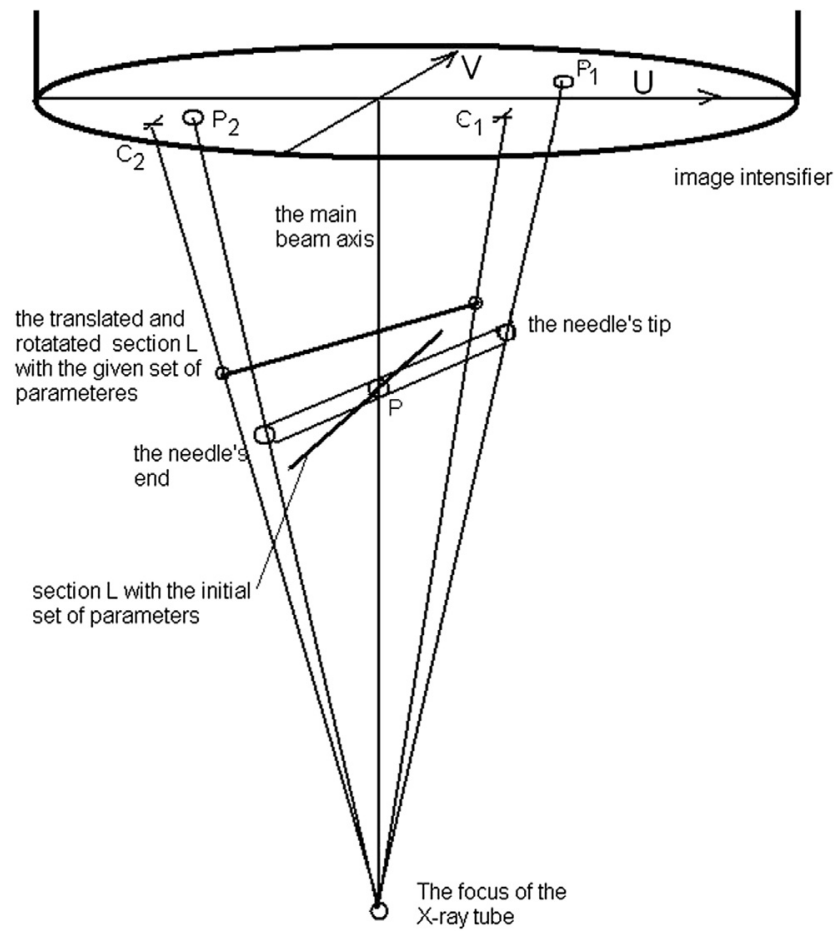

Figure 2 Projections of a needle's tip and end (points $P_{1}$ and $P_{2}$ ) and the projections of tip and end of translated and rotated section $L$ (points $C_{1}$ and $C_{2}$ ) on the PA reconstruction image in course of the multi-parametric fit. points ( $P 1$ and $P 2$ in Fig. 2) can be the tip and the end of the needle, or two radio-opaque markers inserted into the needle being out of the target volume. The distance between the two points is known $(\mathrm{L})$. The spatial position of a BT needle is described with a coordinate system fixed to the isocenter by three translational and three rotational parameters. To incorporate the determination of the magnification factors into the process, the computation of spatial position of BT needle being at the main beam axis is performed with two additional fit parameters of the magnification factors. From the initial values of parameters with a given set of parameters the translated and rotated position of section $L$ is computed. With the approximate values of the magnification factors, the projections of the tip and end of section $L$ (points $C 1$ and $C 2$ in Fig. 2) are generated and the distance between the points $\mathrm{C} 1$ and $\mathrm{P} 1$ and $\mathrm{C} 2$ and $\mathrm{P} 2$ is evaluated. After small variation of the parameters' values the process is repeated until match of the projected tip and end of section $L$ and the digitized points is achieved. Since the coordinates of P1 and P2 involve errors due to the limited pixel size of the images and the imperfect fit of the radio-opaque markers in the needle, therefore exact match of the digitized and computed projected points does not occur. The minima of these distances represent the best approximation of the parameters.

For computation of the projection of the tip and end of section $L$ with actual parameters values, several data are needed: the imaging setup parameters: the distance
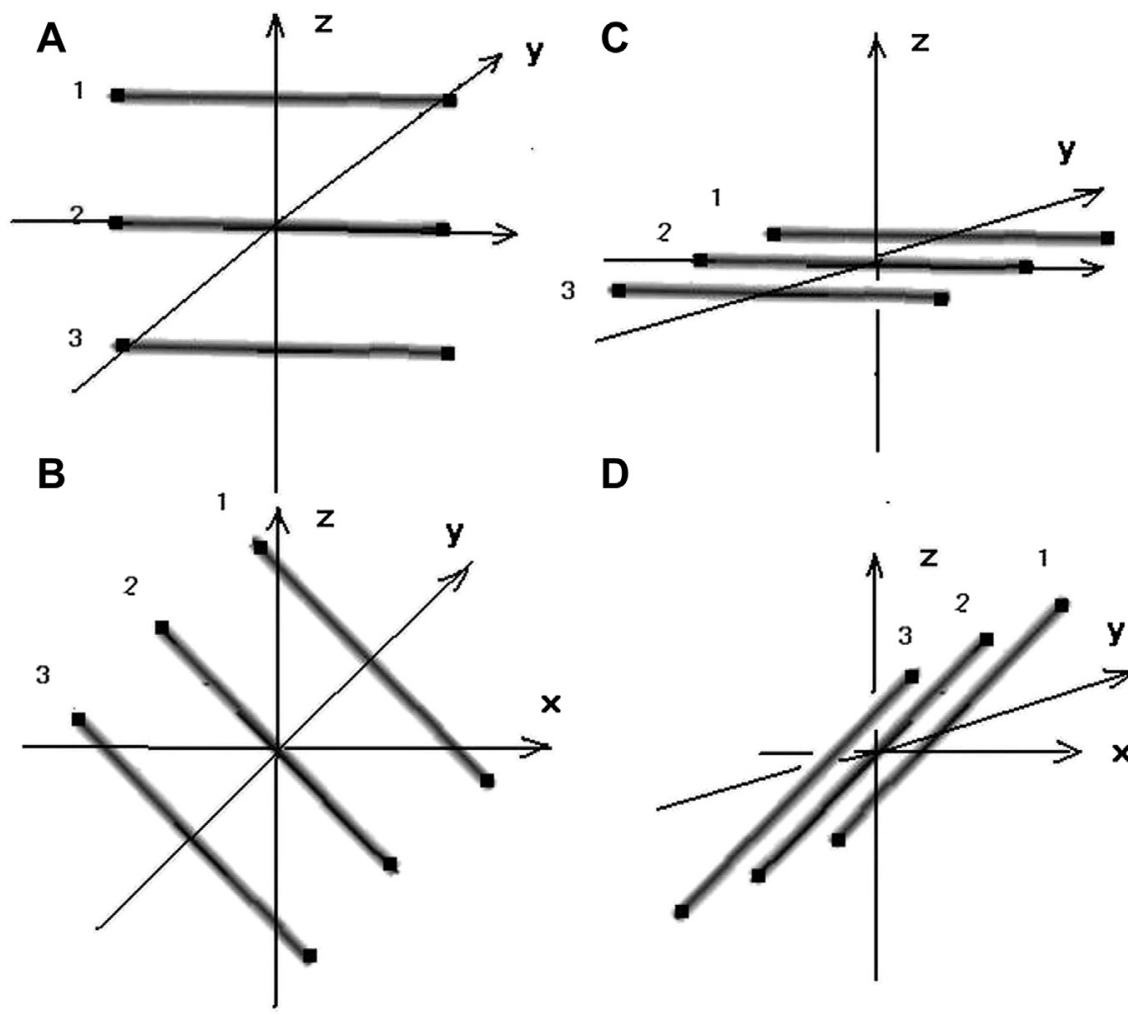

D

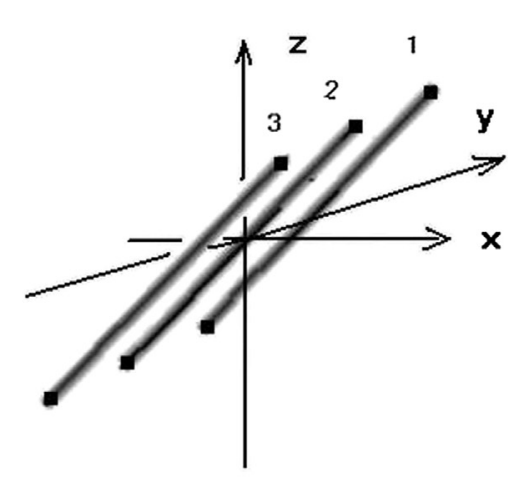

Figure 3 Simulation of BT needles with radio-opaque markers representing the tips and end points positioned into a vertical plane $(A)$ and into a horizontal plane $(C)$ and with rotated needles' plane through $45^{\circ}(B$ and $D)$. 
between the X-ray tube's focus and the image intensifier, the reconstruction angles and the calibrated pixel size of the reconstruction images. The MPF fit requires initial values of the parameters to be fitted obtained as follows. First, the needle located at the main beam axis (\#3 in Fig. 1) is reconstructed. The initial values of the three translational and three rotational parameters represent an imaginary needle being at the isocenter. The middle of the needle is located at the isocenter and directed to the approximate angle of the needle to be reconstructed, as illustrated in Fig. 2.

The MPF program reads the digitized coordinates of the radio-opaque markers, the initial coordinates of the needle to be fitted, the imaging setup data and computes the best approximation of the three translational and three rotational parameters and the magnification factors with minimizing the distances between points $\mathrm{P} 1$ and $\mathrm{C} 1$ and P2 and $\mathrm{C} 2$ using the simplex fit method [7]. The reconstruction of the needle being at the isocenter results in the exact values of the magnification factors of the reconstruction images. The spatial position of the BT needles being out of the mean beam axis is computed by fitting with three translational and with three rotational parameters and with the known magnification factors. The initial parameters for MPF of the needles located out of the isocenter are taken from the previously reconstructed neighboring needle's parameters (\#3 $\rightarrow \# 2$, \#2 $\rightarrow \# 1, \# 3 \rightarrow \# 4$, \#4 $\rightarrow \# 5$ in Fig. 1).

The main factors that influence the reconstruction accuracy are as follows: the limited range of reconstruction angles caused by the treatment table's shield and the lithotomic position of the patient, the possible needles overlap, the imperfect fit of radio-opaque markers in the needle, the pixel size of the images, the digitization error from the screen with a mouse and the distortion of the image intensifier.

The MPF reconstruction accuracy was tested with small circular ( $\varnothing 2 \mathrm{~mm}$ ) radio-opaque markers placed into known position in a single plane representing the tip and end points of three needles being parallel in a distance of $21.2 \mathrm{~mm}$. The two types of insertions with free hand technique are: the treatments of surface malignancies with needles inserted at a horizontal plane and treatments like the carcinoma of the vulva with needles inserted into the labia majora, as illustrated in Fig. 1. We simulated the two types of treatments with positioning the markers' plane horizontally and vertically and series of reconstruction images was obtained between $150^{\circ}$ and $205^{\circ}$. To prevent the markers' overlap, we set the reconstruction angle instead of $180^{\circ}-187.5^{\circ}$. We performed MPF reconstructions with different images pairs. Since the needles are usually not perpendicular to the main beam axis, we repeated the investigation with rotated markers' plane through $45^{\circ}$ as illustrated in Fig. 3. The dose distribution of the BT needles located parallel on a single plane with the same active length is influenced primarily by the distance between the needles. We computed therefore the distance between the simulated needles and the difference between the reconstructed and expected values, listed in Table 1.

We also tested applicability and reproducibility of the MPF method with 9 treatment fractions of three vulvar cancer patients. The insertions were performed with free
Table 1 Difference of the reconstructed and expected distance between the simulated needles (difference 1 and difference 2) represented by six radio-opaque markers placed in a single plane. The markers' plane was located vertically and horizontally. The test was repeated with the needles' plane rotated through $45^{\circ}$. MPF reconstructions were performed with different image pairs.

\begin{tabular}{lll}
\hline Pair of reconstruction & Difference & Difference \\
angles (degrees) & $1(\mathrm{~mm})$ & $2(\mathrm{~mm})$ \\
\hline
\end{tabular}

The position of the radio-opaque plane: vertical No rotation

$\begin{array}{lll}187.5-150 & -0.8 & -0.2 \\ 195-165 & 0.9 & -1.2 \\ 195-150 & -0.5 & 0.0 \\ 205-150 & -1.2 & -0.4\end{array}$

The position of the radio-opaque plane: vertical

$\begin{array}{lll}\text { Rotation angle } & 45^{\circ} & \text { B in Fig. 3 } \\ 187.5-150 & 0.2 & -0.3 \\ 195-165 & -1.3 & -0.2 \\ 195-150 & -0.5 & 0.1 \\ 205-150 & 0.7 & 0.3\end{array}$

The position of the radio-opaque plane: horizontal No rotation

C in Fig. 3

$\begin{array}{lll}180-150 & -0.2 & -0.4 \\ 195-165 & -0.2 & -0.2 \\ 195-150 & 0.1 & -0.4 \\ 205-150 & -0.1 & 0.2\end{array}$

The position of the radio-opaque plane: horizontal

\begin{tabular}{cll} 
Rotation angle & $45^{\circ}$ & D in Fig. 3 \\
$180-150$ & 0.3 & 0.7 \\
$195-165$ & 0.8 & 0.9 \\
$205-150$ & 0.6 & -0.2 \\
$195-150$ & -0.8 & -0.7 \\
\hline
\end{tabular}

hand technique. The number of the needles varied between 3 and 5 , they were inserted into the inner side of the labia majora and the distance between the needles was approximately $1 \mathrm{~cm}$. We obtained more reconstruction images per fraction and the needles were reconstructed with different image pairs. The tips' coordinates obtained with image pairs of PA and $150^{\circ}$ were selected for reference, or in two fractions, to prevent the needles' overlap we used the closest angles to these values. The difference between the reconstructed needles' tip coordinates and those obtained with the reference image pairs was computed.

\section{Results}

The error of the reconstructed end expected distances between the simulated needles varied between $-1.3 \mathrm{~mm}$ and $0.9 \mathrm{~mm}$. No significant difference was given for these values with different image pairs, when the difference between the reconstruction angles was at least $30^{\circ}$. Results of reconstruction of 9 vulvar treatment fractions of three patients with different image pairs are summarized in Table 2. The majority of the needles' tip coordinates agreed to those obtained with the reference image pairs within $3 \mathrm{~mm}$. 
Table 2 The three-dimensional difference in reconstructed needles' tip coordinates (\#1...\#5) obtained with different image pairs compared to ones obtained with the reference image pairs in vulvar insertions.

\begin{tabular}{|c|c|c|c|c|c|c|c|c|c|}
\hline \multirow[t]{2}{*}{$\begin{array}{l}\text { Patient \# } \\
\text { Fraction \# }\end{array}$} & \multirow[t]{2}{*}{$\begin{array}{l}\text { Reference image pair } \\
\text { angles (degrees) }\end{array}$} & \multirow[t]{2}{*}{$\begin{array}{l}\text { Image pair angles } \\
\text { (degrees) }\end{array}$} & \multicolumn{5}{|c|}{$\begin{array}{l}\text { 3D difference in tips' } \\
\text { coordinates }(\mathrm{mm})\end{array}$} & \multirow[t]{2}{*}{$\begin{array}{l}\text { Average } \\
(\mathrm{mm})\end{array}$} & \multirow[t]{2}{*}{$\begin{array}{l}\text { Standard } \\
\text { deviation }(\mathrm{mm})\end{array}$} \\
\hline & & & $\# 1$ & $\# 2$ & $\# 3$ & $\# 4$ & $\# 5$ & & \\
\hline \multirow[t]{4}{*}{1.1} & 180 & 195 & & & & & & & \\
\hline & 157.5 & 157.5 & 1.0 & 1.2 & 1.0 & & & 1.1 & 1.3 \\
\hline & & 195 & & & & & & & \\
\hline & & 165 & 2.6 & 2.6 & 0.7 & & & 2.0 & 1.1 \\
\hline \multirow[t]{4}{*}{1.2} & 180 & 195 & & & & & & & \\
\hline & 150 & 150 & 2.0 & 0.9 & 2.3 & & & 1.7 & 0.7 \\
\hline & & 195 & & & & & & & \\
\hline & & 165 & 3.2 & 0.7 & 1.7 & & & 1.9 & 1.3 \\
\hline \multirow[t]{4}{*}{1.3} & 180 & 195 & & & & & & & \\
\hline & 150 & 150 & 2.2 & 1.3 & 1.0 & 1.0 & 0.9 & 1.3 & 0.2 \\
\hline & & 195 & & & & & & & \\
\hline & & 65 & 2.9 & 2.2 & 1.5 & 1.3 & 0.7 & 1.7 & 0.8 \\
\hline \multirow[t]{2}{*}{1.4} & 180 & 195 & & & & & & & \\
\hline & 150 & 150 & 1.3 & 0.9 & 0.5 & & & 0.9 & 0.3 \\
\hline \multirow[t]{2}{*}{2.1} & 180 & 180 & & & & & & & \\
\hline & 150 & 157.5 & 1.9 & 1.2 & 0.6 & 1.1 & 1.4 & 1.2 & 0.5 \\
\hline \multirow[t]{4}{*}{2.2} & 180 & 205 & & & & & & & \\
\hline & 150 & 150 & 1.4 & 3.3 & 3.9 & & & 2.9 & 1.3 \\
\hline & & 205 & & & & & & & \\
\hline & & 165 & 2.2 & 3.0 & 2.1 & & & 2.4 & 0.5 \\
\hline \multirow[t]{4}{*}{2.3} & 180 & 180 & & & & & & & \\
\hline & 150 & 142.5 & 2.1 & 1.6 & 1.5 & 2.1 & & 1.8 & 0.3 \\
\hline & & 195 & & & & & & & \\
\hline & & 150 & 1.3 & 2.1 & 0.8 & 2.0 & & 1.6 & 0.6 \\
\hline \multirow[t]{2}{*}{2.4} & 180 & 180 & & & & & & & \\
\hline & 150 & 142.5 & 1.2 & 1.3 & 0.6 & 1.5 & & 1.2 & 0.4 \\
\hline \multirow[t]{2}{*}{3.1} & 172.5 & 172 & & & & & & & \\
\hline & 150 & 142.5 & 2.3 & 0.9 & 0.9 & & & 1.4 & 0.8 \\
\hline
\end{tabular}

\section{Discussion and conclusion}

The accuracy of the variable angle reconstruction method with an IBU was investigated by Kolkmann-Deurloo et al. [8], [9]. The applicator reconstruction was accurate for objects being near the isocenter with reconstruction angles set symmetrically to the zero degree with a difference larger than $40^{\circ}$. The accuracy rapidly decreased, when the difference between the two angles was smaller than $25^{\circ}$. Previously, we experienced an acceptable accuracy in the Fletcher-Suit-Delclos applicator reconstruction with C-arm imaging and the Plato Brachytherapy Planning System v13.7 (Nucletron, The Netherlands) [5] and also with MPF fit [6]. The reconstruction image pairs were PA and PO angles between $135^{\circ}$ and $150^{\circ}$. The imaging setup that resulted in an accurate reconstruction of $\mathrm{BT}$ needles with pairs of reconstruction angles with larger difference of $30^{\circ}$ between them.

Using online fluoroscopy images, the reconstruction accuracy is also influenced by the distortion of the image intensifier and a distortion correction is needed [4,9]. We used a small diameter $(16 \mathrm{~cm})$ image intensifier for taking the reconstruction images that showed low S-distortion and limited pincushion distortion at the image corners. With our method for taking images, the majority of the needles appear on the low distortion region of the image intensifier and the needle used for the determination of the magnification factors is always at the center of the image pairs [5]. However, generally the complex dependence of the image intensifier's distortion with the C-arm angle can decrease the accuracy.

The advantage of the MPF method is that it gets around the drawback of the $\mathrm{C}$-arm imaging and it incorporates the determination of the magnification factors of the reconstruction images in applicator reconstruction. The BT treatments are performed similar to the IBU. The patient and the BT needles remain in the same position during the treatment planning and during the irradiation. Although, the reconstruction accuracy of the MPF method based on the C-arm imaging is lower compared to the IBU imaging and conventional biplane reconstruction, it has been found suitable for clinical use.

\section{Acknowledgment}

The author wishes to thank Kofi Agemang Prempeh M.D. and Adel Ambrus M.D. for performing the vulvar insertions for the study. 


\section{References}

[1] Kavanagh BD, Zwicker RD, Segreti EM, Lindquist LA, Fulcher AS, Hundley GM, et al. Gynecologic brachytherapy: digital fluoroscopy for placement verification and treatment planning. Radiology 2000;215:900-3.

[2] Cuipers JP. Clinical use of a non-isocentric C-arm unit for online filmless reconstruction of applicators. Radiother Oncol 2001;61:203-9.

[3] Fung YC. C-arm imaging for brachytherapy source reconstruction: geometrical accuracy. Med Phys 2002;29:724-6.

[4] Liu L, Bassano DA, Prasad SC, Keshler BLK, Hahn SS. On the use of $\mathrm{C}$-arm fluoroscopy for treatment planning in high dose rate brachytherapy. Med Phys 2003;30:2297-302.
[5] Palvolgyi J. To what extent can digital images obtained with a nonisocentric C-arm be used for brachytherapy treatment planning in gynaecology. Radiother Oncol 2003;67:107-12.

[6] Palvolgyi J. "Multiparametric fit method in reconstruction of Fletcher-Suite-Delclos applicator". Med Phys 2006;33(1): 69-75.

[7] Nelder JA, Mead R. A simplex method for function minimization. Comput J 1965;7:308-13.

[8] Kolkman-Deurloo IKK, Visser AG, Idzes MHM, Levendag PC. Reconstruction accuracy of a dedicated localizer for filmless planning intra-operative brachytherapy. Radiother Oncol 1997; 44:73-81.

[9] Kolkman-Deurloo IKK, de Kruijf WJM, Levendag PC. On-line implant reconstruction in HDR brachytherapy. Radiother Oncol 2006;78:53-9. 